\title{
Toward the Exact WKB Analysis for Higher-Order Painlevé Equations - The Case of Noumi-Yamada Systems ${ }^{\dagger}$
}

\author{
By \\ Yoshitsugu TAKEI*
}

\begin{abstract}
As the first step toward the exact WKB analysis for higher-order Painlevé equations, we study the Stokes geometry of the Noumi-Yamada system. It is shown that there are intriguing relations, similar to those for traditional Painlevé equations, between the Stokes geometry of the Noumi-Yamada system and that of its underlying Lax pair.
\end{abstract}

\section{$\S 1$. Introduction}

In a series of papers [KT1], [AKT], [KT2] (cf. [T1] also) we develop the exact WKB analysis for Painlevé equations with a large parameter. Among the results obtained in this series of papers the most important one is the following: There exist intriguing relations between the Stokes geometry of Painlevé equations and that of the underlying linear equations and, making full use of these geometric relations, one can prove that each two parameter solution of the $J$-th Painlevé equation $\left(P_{J}\right)(J=\mathrm{I}, \mathrm{II}, \ldots, \mathrm{VI})$ can be locally transformed to a solution of the first Painlevé equation $\left(P_{\mathrm{I}}\right)$ near its simple turning point. In this paper and series of our forthcoming articles we want to discuss the generalization of the exact WKB analysis to higher-order Painlevé equations.

Communicated by T. Kawai. Received October 30, 2003.

2000 Mathematics Subject Classification(s): 34M60, 34M55, 34E20, 33E17

Supported by JSPS Grant-in-Aid (No. 13640167 and No. 14340042) and by JSPS JapanAustralia Research Cooperative Program.

${ }^{\dagger}$ This article is an invited contribution to a special issue of Publications of RIMS commemorating the fortieth anniversary of the founding of the Research Institute for Mathematical Sciences.

*RIMS, Kyoto University, Kyoto 606-8502, Japan. 
Let us first explain the general formulation of the problem. Although single differential equations were used as underlying linear equations in [KT1] etc., we use matrix form of equations here. We thus start with the following $N \times N$ system of (formal) linear ordinary differential equations with a large parameter $\eta$ :

$$
\begin{cases}\frac{\partial}{\partial x} \psi=\eta A \psi, & A=A(x, t, u ; \eta)=\sum_{k=0}^{\infty} \eta^{-k} A_{k}(x, t, u), \\ \frac{\partial}{\partial t} \psi=\eta B \psi, & B=B(x, t, u ; \eta)=\sum_{k=0}^{\infty} \eta^{-k} B_{k}(x, t, u),\end{cases}
$$

where $x$ denotes an independent variable, $t$ a deformation parameter, $\psi$ a vector-valued unknown function, and $u=\left\{u_{j}\right\}_{j \in J}$ a tuple of parameters with the index set $J$. That is, we consider a system of first-order linear ordinary differential equations in the $x$ variable (i.e., the first equation of (1.1)) whose deformation in the $t$ direction is governed also by a system of first-order differential equations (the second equation of (1.1)) of the same kind. We now assume that the compatibility condition

$$
\frac{\partial A}{\partial t}-\frac{\partial B}{\partial x}+\eta(A B-B A)=0
$$

of (1.1) is satisfied so that (1.1) may describe an isomonodromic deformation of its first member (i.e., the equation in the $x$ variable). In particular, what we are interested in is the case where the compatibility condition (1.2) is equivalent to (a system of) nonlinear differential equations for the parameters $u=\left\{u_{j}\right\}_{j \in J}$ containing the large parameter $\eta$ :

$$
F_{l}\left(t, u_{j}, \ldots, d^{m} u_{j} / d t^{m} ; \eta\right)=0 \quad(l \in L) .
$$

As is shown in [JMU], all traditional Painlevé equations appear in this context and hence we call (1.3) a (higher-order) Painlevé equation (or, Painlevé type equation) and (1.1) its underlying Lax pair. The "exact WKB analysis for higher-order Painlevé equations" aims at analyzing the (global) behavior of solutions of a Painlevé type equation (1.3) with the help of the exact WKB analysis for its underlying Lax pair (1.1).

In this paper, as the first step of the research in this direction, we study the Stokes geometry of an example of higher-order Painlevé equations proposed by Noumi and Yamada ([NY1], [NY2]). The Noumi-Yamada systems, denoted by $(N Y)_{l}(l=2,3,4, \ldots)$ in what follows, are discovered by Noumi and Yamada through the study of the symmetry of Painleve equations and possess the affine 
Weyl group symmetry of type $A_{l}^{(1)}$. Their explicit form is given as follows: In the case of type $A_{2 m}^{(1)}$ (i.e., when $l=2 m$ ),

$$
\frac{d u_{j}}{d t}=\eta\left[u_{j}\left(u_{j+1}-u_{j+2}+\cdots-u_{j+2 m}\right)+\alpha_{j}\right]
$$

$(j=0,1, \ldots, 2 m)$ where $\alpha_{j}$ are complex parameters satisfying

$$
\alpha_{0}+\cdots+\alpha_{2 m}=\eta^{-1}
$$

and the independent variable $t$ and the unknown functions $u_{j}$ are normalized so that

$$
u_{0}+\cdots+u_{2 m}=t
$$

may be satisfied. On the other hand, in the case of type $A_{2 m+1}^{(1)}$ (i.e., when $l=2 m+1)$,

$$
\begin{aligned}
& (N Y)_{2 m+1} \\
& \frac{t}{2} \frac{d u_{j}}{d t}=\eta\left[u_{j}\left(\sum_{1 \leq r \leq s \leq m} u_{j-1+2 r} u_{j+2 s}-\sum_{1 \leq r \leq s \leq m} u_{j+2 r} u_{j+1+2 s}\right)+\frac{t}{2} \alpha_{j}\right]
\end{aligned}
$$

$(j=0,1, \ldots, 2 m+1)$ where, instead of (1.4) and (1.5), we assume the following in this case:

$$
\begin{aligned}
& \alpha_{0}+\alpha_{2}+\cdots+\alpha_{2 m}=\alpha_{1}+\alpha_{3}+\cdots+\alpha_{2 m+1}=\eta^{-1} / 2, \\
& u_{0}+u_{2}+\cdots+u_{2 m}=u_{1}+u_{3}+\cdots+u_{2 m+1}=t / 2 .
\end{aligned}
$$

(In both cases we also assume that $\alpha_{j}$ and $u_{j}$ are cyclic with respect to the index $j$ with the cycle $N=l+1$.) These systems $(N Y)_{l}$ describe the compatibility condition of the following Lax pair of the size $N \times N(N=l+1)$ :

$$
\begin{aligned}
\frac{\partial}{\partial x} \psi & =\eta A \psi, \\
\frac{\partial}{\partial t} \psi & =\eta B \psi,
\end{aligned}
$$

where

$$
A=-\frac{1}{x}\left(\begin{array}{ccccc}
\epsilon_{1} & u_{1} & 1 & & \\
& \ddots & \ddots & \ddots & \\
& & \epsilon_{N-2} & u_{N-2} & 1 \\
x & & & \epsilon_{N-1} & u_{N-1} \\
x u_{0} & x & & & \epsilon_{N}
\end{array}\right)
$$


and

$$
B=\left(\begin{array}{ccccc}
q_{1} & -1 & & & \\
& q_{2} & -1 & & \\
& & \ddots & \ddots & \\
& & & q_{N-1}-1 \\
& & & & q_{N}
\end{array}\right) .
$$

Here $\epsilon_{j}$ are parameters determined by the relations $\alpha_{j}=\epsilon_{j}-\epsilon_{j+1}+\eta^{-1} \delta_{j, 0}$ and $\epsilon_{1}+\cdots+\epsilon_{N}=0\left(\delta_{j, k}\right.$ denotes Kronecker's symbol $)$, and $q_{j}=q_{j}(t)$ are functions of $t$ satisfying $q_{j+2}-q_{j}=u_{j}-u_{j+1}$ and $q_{1}+\cdots+q_{N}=-t / 2$. More explicitly, we take $q_{j}$ as

$$
\begin{aligned}
q_{j} & =u_{j+1}+u_{j+3}+\cdots+u_{j+2 m-1}-\frac{t}{2} \\
& =-\frac{1}{2}\left(u_{j}-u_{j+1}+u_{j+2}-\cdots+u_{j+2 m}\right)
\end{aligned}
$$

in the case of $l=2 m$, and as

$$
\text { (1.13) } \begin{aligned}
q_{j} & =\frac{2}{t} \sum_{1 \leq r \leq s \leq m} u_{j-1+2 r} u_{j+2 s}-\frac{t}{4} \\
& =-\frac{1}{t}\left[\sum_{r=0 \text { or } 0 \leq s<r \leq m} u_{j-1+2 r} u_{j+2 s}-\sum_{1 \leq r \leq s \leq m} u_{j-1+2 r} u_{j+2 s}\right]
\end{aligned}
$$

in the case of $l=2 m+1$. Note that a large parameter $\eta$ has been already introduced into our nonlinear equation $(N Y)_{l}$ together with its underlying Lax pair (1.8) and (1.9) so that we may develop the WKB analysis for them. (If we put $\eta=1$, the original Noumi-Yamada system and its underlying Lax pair are obtained. We have introduced a large parameter into them by an appropriate scaling of the variables.) It is well-known that the first member $(N L)_{2}$ and the second member $(N L)_{3}$ of $(N Y)_{l}$ are equivalent to the traditional Painlevé equations $\left(P_{\mathrm{IV}}\right)$ and $\left(P_{\mathrm{V}}\right)$ respectively. In this sense the Noumi-Yamada systems can be regarded as higher-order analogue of the fourth and fifth Painlevé equations.

The purpose of this paper is to show that the relations of the Stokes geometries similar to those for traditional Painlevé equations also hold for the Noumi-Yamada system $(N Y)_{l}$ and its underlying Lax pair (1.8) and (1.9). The same problem for other examples of higher-order Painlevé equations $\left(\left(P_{\mathrm{I}}\right)\right.$ and $\left(P_{\mathrm{II}}\right)$ hierarchies) will be discussed in [KKNT]. 
Now the plan of this paper is as follows: We give the precise formulation of our main theorems (i.e., the relations of the Stokes geometries) in Section 2. The proof of the main theorems will be done in the subsequent two sections (Sections 3 and 4). Finally in Section 5 we make concluding remarks on some open problems of the exact WKB analysis for higher-order Painlevé equations.

\section{$\S 2 . \quad$ Main Results}

Our equation $(N Y)_{l}$ contains the large parameter $\eta$. Using this structure, we first construct a formal solution of $(N Y)_{l}$ in the following manner: We substitute the formal power series expansion (in $\left.\eta^{-1}\right) u_{j}=u_{j, 0}(t)+\eta^{-1} u_{j, 1}(t)+$ $\cdots(0 \leq j \leq l)$ of unknown functions into the equation and compare like powers of $\eta$. Then we obtain

$$
V_{j}\left(u_{0,0}, u_{1,0}, \ldots, u_{l, 0}\right)=0 \quad(j=0, \ldots, l)
$$

and

$$
\left.\frac{\partial\left(V_{0}, \ldots, V_{l}\right)}{\partial\left(u_{0}, \ldots, u_{l}\right)}\right|_{u_{j}=u_{j, 0}} U_{k}=F_{k} \quad(k=1,2, \ldots) .
$$

Here $U_{k}$ denotes ${ }^{t}\left(u_{0, k}, u_{1, k}, \ldots, u_{l, k}\right), F_{k}$ is an $(l+1)$-vector whose entry is a (recursively determined) differential polynomial of $\left\{u_{j, 0}, u_{j, 1}, \ldots, u_{j, k-1}\right\}_{0 \leq j \leq l}$, and $V_{j}$ denotes a polynomial of $\left(u_{0}, \ldots, u_{l}\right)$ defined by

$$
V_{j}=u_{j}\left(u_{j+1}-u_{j+2}+\cdots-u_{j+2 m}\right)+\alpha_{j}
$$

in the case of $l=2 m$ and by

$$
V_{j}=u_{j}\left(\sum_{1 \leq r \leq s \leq m} u_{j-1+2 r} u_{j+2 s}-\sum_{1 \leq r \leq s \leq m} u_{j+2 r} u_{j+1+2 s}\right)+\frac{t}{2} \alpha_{j}
$$

in the case of $l=2 m+1$ respectively. Thus for each solution $\left(\widehat{u}_{0,0}, \widehat{u}_{1,0}, \ldots, \widehat{u}_{l, 0}\right)$ of the algebraic equation (2.1) we can obtain a (multi-valued) formal solution

$$
\widehat{u}_{j}=\widehat{u}_{j}(t, \eta)=\widehat{u}_{j, 0}(t)+\eta^{-1} \widehat{u}_{j, 1}(t)+\cdots
$$

of $(N Y)_{l}$ outside the set

$$
\left\{t \in \mathbb{C} ; \partial\left(V_{0}, \ldots, V_{l}\right) /\left.\partial\left(u_{0}, \ldots, u_{l}\right)\right|_{u_{j}=\widehat{u}_{j, 0}(t)}=0\right\} .
$$

The formal solution $\widehat{u}=\left\{\widehat{u}_{j}\right\}$ is often called a "0-parameter solution". 
Remark. The system of algebraic equations (2.1) is expected to be 'regular', that is, (2.1) is expected to have (a finite number of) solutions for a generically given $t$. We have confirmed this for $l=2,3,4,5$ by eliminating variables and computing the resultant of (2.1) explicitly. It is, however, still an open problem to prove it for any $l$. In what follows we use the 0-parameter solution $\widehat{u}$ to define the Stokes geometry of $(N Y)_{l}$ and its underlying Lax pair, assuming the existence of $\widehat{u}$, i.e., the 'regularity' of (2.1).

Here let us recall the definition of the Stokes geometry of a system of first-order linear ordinary differential equations.

Definition 2.1. $\quad$ (i) For a system of linear differential equations

$$
\frac{d}{d x} \psi=\eta A \psi, \quad A=A(x, \eta)=A_{0}(x)+\eta^{-1} A_{1}(x)+\cdots,
$$

we call a zero of the discriminant of the characteristic equation of $A_{0}(x)$ a turning point of (2.7). That is, $x=x_{0}$ is a turning point if and only if there exist two eigenvalues $\lambda_{n}(x)$ and $\lambda_{n^{\prime}}(x)$ of $A_{0}(x)$ which merge at $x=x_{0}$. In particular, a simple (resp. double) zero of the discriminant is called a simple (resp. double) turning point.

(ii) We call a (real one-dimensional) curve defined by the following relation (2.8) a Stokes curve of (2.7):

$$
\operatorname{Im} \int_{x_{0}}^{x}\left(\lambda_{n}(x)-\lambda_{n^{\prime}}(x)\right) d x=0,
$$

where $\lambda_{n}(x)$ and $\lambda_{n^{\prime}}(x)$ are two eigenvalues of $A_{0}(x)$ which merge at a turning point $x=x_{0}$.

We then define the Stokes geometry of the Noumi-Yamada system $(N Y)_{l}$ by using the 0-parameter solution as follows:

Definition 2.2. $\quad$ A turning point (resp. Stokes curve) of $(N Y)_{l}$ is, by definition, a turning point (resp. Stokes curve) of the linearized equation (i.e., the first variational equation) of $(N Y)_{l}$ at a 0 -parameter solution $\widehat{u}=\left\{\widehat{u}_{j}\right\}$.

Note that the linearized equation of $(N Y)_{l}$ at $\widehat{u}$ can be obtained by setting $u_{j}=\widehat{u}_{j}+\Delta u_{j}$ in $(N Y)_{l}$ and by taking its linear part in $\left\{\Delta u_{j}\right\}$. Since the equation thus obtained is a system of first-order linear differential equations (in the variable $t$ ) with the unknown function $\Delta u={ }^{t}\left(\Delta u_{0}, \ldots, \Delta u_{l}\right)$, its Stokes geometry is defined by Definition 2.1 (with replacing the variable $x$ there by $t$ ). 
Substituting the 0-parameter solution (2.5) into the coefficients of the underlying Lax pair (1.8) and (1.9), we obtain the Lax pair

$$
\begin{aligned}
\frac{\partial}{\partial x} \psi & =\eta A \psi, \quad A=A(x, t ; \eta)=A_{0}(x, t)+\eta^{-1} A_{1}(x, t)+\cdots \\
\frac{\partial}{\partial t} \psi & =\eta B \psi, \quad B=B(x, t ; \eta)=B_{0}(x, t)+\eta^{-1} B_{1}(x, t)+\cdots
\end{aligned}
$$

the compatibility condition of which is satisfied as a formal power series of $\eta^{-1}$. On the other hand, let

$$
\frac{\partial}{\partial t} \Delta u=\eta C \Delta u, \quad C=C(t ; \eta)=C_{0}(t)+\eta^{-1} C_{1}(t)+\cdots
$$

be the linearized equation of $(N Y)_{l}$ at $\widehat{u}$. Our problem is now to study the relations between the Stokes geometry of (2.11) (that is, the Stokes geometry of the Noumi-Yamada system $\left.(N Y)_{l}\right)$ and that of the underlying Lax pair (2.9) and (2.10) (especially, that of the first equation (2.9) with viewing $t$ as a deformation parameter).

We first observe the following intriguing relations concerning the top order part $A_{0}, B_{0}$ and $C_{0}$.

Proposition 2.1. Let $D_{A_{0}}(x, t)$ and $D_{B_{0}}(x, t)$ denote the discriminant of the characteristic equations $\operatorname{det}\left(\lambda-A_{0}(x, t)\right)=0$ and $\operatorname{det}\left(\mu-B_{0}(x, t)\right)=0$ respectively. We also let $D(x, t)$ denote

$$
D(x, t)=\prod_{1 \leq n<n^{\prime} \leq N}\left(\mu_{n}+\mu_{n^{\prime}}\right),
$$

where $\mu_{n}$ are eigenvalues of $B_{0}$. Then $D(x, t)$ is a polynomial of degree at most $m$ in $x$ (whose coefficients are analytic functions of $t$ ) and the following relation holds:

$$
D_{A_{0}}(x, t)=x^{-N(N-1)} D(x, t)^{2} D_{B_{0}}(x, t) .
$$

Thus, zeroes of $D(x, t)$ provide (generically) double turning points of the system (2.9) and zeroes of $D_{B_{0}}(x, t)$ (i.e., turning points of (2.10)) provide its (generically) simple turning points.

Remark. The discriminant $D_{B_{0}}(x, t)$ is a polynomial of degree $l=N-1$ in $x$, and hence there are $l$ simple turning points. We can also verify that the degree of $D(x, t)$ is exactly equal to $m$ in the case of $l=2 m$. 
Proposition 2.2. Letting $\lambda_{n}(x, t)$ and $\mu_{n}(x, t)(1 \leq n \leq N)$ respectively denote eigenvalues of $A_{0}$ and $B_{0}$, we find

$$
\frac{\partial}{\partial t} \lambda_{n}(x, t)=\frac{\partial}{\partial x} \mu_{n}(x, t) \quad(n=1, \ldots, N) .
$$

Proposition 2.3. We decompose the characteristic equation of $B_{0}$ into the sum of its odd-degree part and even-degree part (with respect to $\mu$ ):

$$
\begin{aligned}
\operatorname{det}\left(\mu-B_{0}(x, t)\right) & =\left(\mu-q_{1,0}\right) \cdots\left(\mu-q_{N, 0}\right)+(-1)^{N-1} x \\
& =g_{\text {odd }}(\mu, t)+g_{\text {even }}(\mu, x, t) .
\end{aligned}
$$

(Here $q_{j, 0}$ denotes the top order part of $q_{j}$. Note that $g_{\text {odd }}(\mu, t)$ does not depend on $x$ in view of (2.15).) Then the following relation holds between the characteristic equation of $C_{0}$ and $g_{\text {odd }}(\mu, t)$ :

$$
\operatorname{det}\left(\nu-C_{0}(t)\right)= \begin{cases}\left.2^{N} g_{\text {odd }}(\mu, t)\right|_{\mu=\nu / 2} & (\text { when } l=2 m), \\ \left.2^{N}\left(\mu \tilde{g}_{\text {odd }}(\mu, t)\right)\right|_{\mu=\nu / 2} & (\text { when } l=2 m+1),\end{cases}
$$

where $N=l+1$ and $\tilde{g}_{\text {odd }}(\mu, t)$ denotes the monic polynomial of $\mu$ obtained by dividing $g_{\text {odd }}(\mu, t)$ by the coefficient of its top degree part. $\left(\right.$ Hence $\tilde{g}_{\text {odd }}=g_{\text {odd }}$ holds in the case of $l=2 \mathrm{~m}$.)

The proof of Proposition 2.1 and that of Proposition 2.3 are respectively given in Sections 3 and 4 below, while Proposition 2.2 is proved in [T2] in a more general context by employing the method of diagonalization for the Lax pair (2.9) and (2.10).

It follows from Proposition 2.3 that the characteristic equation $\operatorname{det}(\nu-$ $\left.C_{0}(t)\right)=0$ of $C_{0}$ has the form $\nu f\left(\nu^{2}, t\right)$ (when $l=2 m$ ) or $\nu^{2} f\left(\nu^{2}, t\right)$ (when $l=2 m+1)$ with some polynomial $f$ of degree $m$. Therefore there are two kinds of turning points for the Noumi-Yamada system $(N Y)_{l}$ : A turning point where the degree 0 part of $f$ vanishes ("a turning point of the first kind"), and a turning point where the discriminant of $f$ vanishes ("a turning point of the second kind"). In what follows we impose the following genericity condition:

$$
\text { At every zero of } D_{B_{0}}(x, t) \text { just two of the eigenvalues of } B_{0}(x, t)
$$
merge and the other eigenvalues are mutually distinct.

Note that the assumption (2.17) guarantees that all turning points of $B_{0}$ are simple as $\partial\left(\operatorname{det}\left(\mu-B_{0}\right)\right) / \partial x$ never vanishes. Then, under the assumption (2.17), by using the above three propositions we can prove the following main theorems for each kind of turning points. 
Theorem 2.1. Let $t=t_{0}$ be a turning point of the first kind of the Noumi-Yamada system $(N Y)_{l}$. Then the following holds.

(i) At $t=t_{0}$ a double turning point (denoted by $x_{2}(t)$ ) of (2.9) (i.e., a zero of $D(x, t)$ in $(2.13)$ ) merges with a simple turning point (denoted by $x_{1}(t)$ ) of (2.9) (i.e., a zero of $D_{B_{0}}(x, t)$ in (2.13)). Furthermore, a pair of the eigenvalues of $A_{0}(x, t)$ merging at $x=x_{1}(t)$ also merges at $x=x_{2}(t)$.

(ii) Let $\lambda^{+}$and $\lambda^{-}$be the two eigenvalues of $A_{0}(x, t)$ merging at $x=x_{1}(t)$ and $x=x_{2}(t)$, and let $\nu^{+}$and $\nu^{-}$be the two eigenvalues of $C_{0}(t)$ satisfying $\nu^{+}\left(t_{0}\right)=\nu^{-}\left(t_{0}\right)=0$ at $t=t_{0}$ and also $\nu^{-}=-\nu^{+}$. Then we find

$$
\frac{1}{2} \int_{t_{0}}^{t}\left(\nu^{+}-\nu^{-}\right) d t=\int_{x_{1}(t)}^{x_{2}(t)}\left(\lambda^{+}-\lambda^{-}\right) d x
$$

In particular, if $t$ lies on a Stokes curve of $(N Y)_{l}$ emanating from $t=t_{0}$, a double turning point $x=x_{2}(t)$ and a simple turning point $x=x_{1}(t)$ are connected by a Stokes curve of (2.9).

Theorem 2.2. Let $t=t_{0}$ be a turning point of the second kind of the Noumi-Yamada system $(N Y)_{l}$. Then the following holds.

(i) At $t=t_{0}$ a double turning point (denoted by $x_{1}(t)$ ) of (2.9) merges with another double turning point (denoted by $x_{2}(t)$ ) of (2.9). Furthermore, a pair of the eigenvalues of $A_{0}(x, t)$ merges both at $x=x_{1}(t)$ and at $x=x_{2}(t)$.

(ii) Let $\lambda^{+}$and $\lambda^{-}$be the two eigenvalues of $A_{0}(x, t)$ merging at $x=x_{1}(t)$ and $x=x_{2}(t)$, and let $\nu_{k}^{ \pm}$and $\nu_{k^{\prime}}^{ \pm}$be the eigenvalues of $C_{0}(t)$ satisfying $\nu_{k}^{+}\left(t_{0}\right)=$ $\nu_{k^{\prime}}^{+}\left(t_{0}\right)$ at $t=t_{0}$ together with $\nu_{k}^{-}=-\nu_{k}^{+}$. Then we find

$$
\int_{t_{0}}^{t}\left(\nu_{k}^{+}-\nu_{k^{\prime}}^{+}\right) d t=-\int_{t_{0}}^{t}\left(\nu_{k}^{-}-\nu_{k^{\prime}}^{-}\right) d t=\int_{x_{1}(t)}^{x_{2}(t)}\left(\lambda^{+}-\lambda^{-}\right) d x .
$$

In particular, if $t$ lies on a Stokes curve of $(N Y)_{l}$ emanating from $t=t_{0}$, two double turning points $x=x_{1}(t)$ and $x=x_{2}(t)$ are connected by a Stokes curve of $(2.9)$.

Theorems 2.1 and 2.2 describe the relations between the Stokes geometry of the Noumi-Yamada system $(N Y)_{l}$ and that of (the first member (2.9) of) its underlying Lax pair. The relation described in Theorem 2.1 is completely the same as that for the traditional Painlevé equations, while the relation in Theorem 2.2 is new: A turning point of the second kind is a new kind of turning points peculiar to higher-order nonlinear equations. The proof of Theorems 2.1 and 2.2 will be given in Section 3 . 


\section{$\S 3 . \quad$ Proof of the Main Results}

In this section we prove Proposition 2.1, Theorem 2.1 and Theorem 2.2. The proof of Proposition 2.3 will be done in the subsequent section (Section $4)$ as it is lengthy. In Sections 3 and 4 , to denote the top order part $u_{j, 0}, q_{j, 0}$, etc., we use the abbreviated notation $u_{j}, q_{j}, \ldots$ as there will be no fear of confusions.

\section{§3.1. Proof of Proposition 2.1}

Take a generic point $(x, t)$ so that $D_{B_{0}}(x, t)$ never vanishes in a neighborhood of it, and let $\mu_{n}$ and $\Phi_{n}$ be an eigenvalue and a corresponding eigenvector of $B_{0}$ there respectively. In view of the explicit form (1.11) of $B_{0}$, we may assume $\Phi_{n}$ is of the form

$$
\Phi_{n}={ }^{t}\left(1,\left(q_{1}-\mu_{n}\right),\left(q_{1}-\mu_{n}\right)\left(q_{2}-\mu_{n}\right), \ldots\right) .
$$

Now, since the Lax pair (2.9) and (2.10) in question satisfies the compatibility condition (1.2), its top order parts $A_{0}$ and $B_{0}$ do commute. This implies that $\Phi_{n}$ is simultaneously an eigenvector of $A_{0}$, that is, $A_{0} \Phi_{n}=\lambda_{n} \Phi_{n}$ holds for some eigenvalue $\lambda_{n}$ of $A_{0}$. In particular, looking at the first entry of the equation $A_{0} \Phi_{n}=\lambda_{n} \Phi_{n}$, we find

$$
\begin{aligned}
\lambda_{n} & =-\frac{1}{x}\left[\epsilon_{1}+u_{1}\left(q_{1}-\mu_{n}\right)+\left(q_{1}-\mu_{n}\right)\left(q_{2}-\mu_{n}\right)\right] \\
& =-\frac{1}{x}\left[\mu_{n}^{2}-\left(q_{1}+q_{2}+u_{1}\right) \mu_{n}+q_{1} q_{2}+u_{1} q_{1}+\epsilon_{1}\right] \\
& =-\frac{1}{x}\left[\left(\mu_{n}-\frac{q_{1}+q_{2}+u_{1}}{2}\right)^{2}-\frac{1}{4}\left(q_{1}+q_{2}+u_{1}\right)^{2}+q_{1} q_{2}+u_{1} q_{1}+\epsilon_{1}\right] .
\end{aligned}
$$

Here let us note the following

Lemma 3.1. Let $w_{j}$ denote $q_{j}+q_{j+1}+u_{j}$. Then $w_{j}=0$ for any $j$.

Proof. It follows from the relation $q_{j+2}-q_{j}=u_{j}-u_{j+1}$ that $w_{j}=w_{j+1}$ holds. Hence $w_{j}$ is independent of $j$ and consequently we have

$$
N w_{j}=2\left(q_{1}+\cdots+q_{N}\right)+\left(u_{0}+\cdots+u_{N-1}\right)=2(-t / 2)+t=0 .
$$

That is, $w_{j}=0$. 
Lemma 3.1 entails

$$
\lambda_{n}=-\frac{1}{x}\left(\mu_{n}^{2}+q_{1} q_{2}+u_{1} q_{1}+\epsilon_{1}\right) .
$$

Since $D_{A_{0}}$ is, by the definition, the square of the difference product of the eigenvalues of $A_{0},(3.4)$ leads to

$$
\begin{aligned}
D_{A_{0}}(x, t) & =\prod_{1 \leq n<n^{\prime} \leq N}\left(\lambda_{n}-\lambda_{n^{\prime}}\right)^{2} \\
& =x^{-N(N-1)} \prod_{\substack{1 \leq n<n^{\prime} \leq N\\
}}\left(\mu_{n}+\mu_{n^{\prime}}\right)^{2} \prod_{1 \leq n<n^{\prime} \leq N}\left(\mu_{n}-\mu_{n^{\prime}}\right)^{2} \\
& =x^{-N(N-1)} D(x, t)^{2} D_{B_{0}}(x, t) .
\end{aligned}
$$

This completes the proof of (2.13).

It follows from the explicit form $(2.15)$ of $\operatorname{det}\left(\mu-B_{0}\right)$ that the product $\mu_{1} \cdots \mu_{N}$ is a linear polynomial of $x$ and that all the other elementary symmetric polynomials of $\left\{\mu_{j}\right\}$ except the product are independent of $x$. Since $D(x, t)$ is a symmetric polynomial of $\left\{\mu_{j}\right\}$ of degree $N(N-1) / 2$, we can conclude that $D(x, t)$ is a polynomial of degree at most $m$ in $x$. Thus all the assertions in Proposition 2.1 are proved.

Remark. Using Sylvester's expression

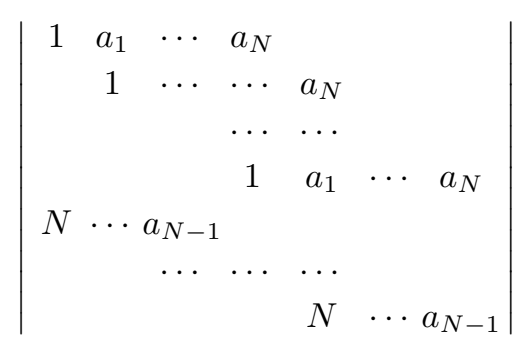

for the resultant between a polynomial $p=\mu^{N}+a_{1} \mu^{N-1}+\cdots+a_{N}$ and its derivative $p^{\prime}=N \mu^{N-1}+\cdots+a_{N-1}$ with $p=\operatorname{det}\left(\mu-B_{0}\right)$, we readily find that the discriminant $D_{B_{0}}(x, t)$ is a polynomial of degree $l=N-1$ in $x$. Furthermore, in the case of $l=2 m$ (i.e., $N=2 m+1$ ), using this observation, we can also verify that $D(x, t)$ is a polynomial of degree exactly equal to $m$ in $x$ in the following manner:

We compute the degree (in $x$ ) of $x^{N(N-1)} D_{A_{0}}(x, t)$. Since the discriminant $D_{A_{0}}(x, t)$ is a symmetric polynomial of the eigenvalues $\left\{\lambda_{n}\right\}$ of $A_{0}$ with degree $N(N-1), x^{N(N-1)} D_{A_{0}}(x, t)$ has an expression

$$
x^{N(N-1)} D_{A_{0}}(x, t)=\sum_{\alpha} c_{\alpha} \sigma_{1}^{\alpha_{1}} \cdots \sigma_{N}^{\alpha_{N}},
$$


where $\sigma_{n}$ denotes the $n$-th elementary symmetric polynomial of $\left\{x \lambda_{n}\right\}$ and the summation runs over a set of the indices satisfying $\alpha_{1}+2 \alpha_{2}+\cdots+N \alpha_{N}=$ $N(N-1)$. Here an explicit computation of $\operatorname{det}\left(x \lambda-x A_{0}\right)$ tells us that $\sigma_{N}=$ $\sigma_{2 m+1}$ is a polynomial of degree 2 in $x, \sigma_{n}$ is a polynomial of degree at most 1 for $m+1 \leq n \leq 2 m$, and $\sigma_{n}$ is independent of $x$ for $1 \leq n \leq m$. This implies that the degree of $x^{N(N-1)} D_{A_{0}}(x, t)$ is at most $\alpha_{m+1}+\cdots+\alpha_{2 m}+2 \alpha_{2 m+1}$, but this can be estimated as

$$
\begin{aligned}
& \frac{1}{2}(2 m+1)\left(\alpha_{m+1}+\cdots+\alpha_{2 m}+2 \alpha_{2 m+1}\right) \\
& =\left(m+\frac{1}{2}\right) \alpha_{m+1}+\cdots+\left(m+\frac{1}{2}\right) \alpha_{2 m}+(2 m+1) \alpha_{2 m+1} \\
& \leq \alpha_{1}+\cdots+(m+1) \alpha_{m+1}+\cdots+2 m \alpha_{2 m}+(2 m+1) \alpha_{2 m+1} \\
& =2 m(2 m+1),
\end{aligned}
$$

where the equality is attained only for $\alpha_{1}=\cdots=\alpha_{2 m}=0, \alpha_{2 m+1}=2 m$. Hence $x^{N(N-1)} D_{A_{0}}(x, t)$ is of degree at most $4 m$ and the term of degree $4 m$ (if it exists) comes only from $\sigma_{2 m+1}^{2 m}$. On the other hand, using Sylvester's expression again for $x^{N(N-1)} D_{A_{0}}(x, t)$, we can confirm that the term $\sigma_{2 m+1}^{2 m}$ does really exist in $x^{N(N-1)} D_{A_{0}}(x, t)$. Thus the degree of $x^{N(N-1)} D_{A_{0}}(x, t)$ is exactly equal to $4 m$ and this together with (2.13) and the above observation leads to the conclusion that the degree of $D(x, t)$ is exactly equal to $m$.

\section{§3.2. Proof of Theorems 2.1 and 2.2}

We first prove Theorem 2.1.

Let $t=t_{0}$ be a turning point of the first kind. Then, thanks to Proposition 2.3 , there exists $\rho(t)$ satisfying

$$
h(\rho(t), t)=0 \quad \text { and } \quad \rho(t) \rightarrow 0\left(\text { as } t \rightarrow t_{0}\right),
$$

where $h(\rho, t)$ is a polynomial of $\rho$ with degree $m(l=2 m$ or $l=2 m+1)$ defined by $g_{\text {odd }}(\mu, t)=\mu h\left(\mu^{2}, t\right)$. In what follows we denote $\sqrt{\rho(t)}$ by $\mu(t)$.

Let us first define $x_{2}(t)$ by the equation

$$
g_{\text {even }}\left(\mu(t), x_{2}(t), t\right)=0 .
$$

Then we readily find that both $\mu(t)$ and $-\mu(t)$ become roots of $\operatorname{det}\left(\mu-B_{0}\right)=0$ at $x=x_{2}(t)$, that is, $\pm \mu(t)$ are eigenvalues of $B_{0}$ there. Hence, by the definition (2.12), $D(x, t)$ vanishes at $x=x_{2}(t)$. This means that $x_{2}(t)$ is a double turning point of $A_{0}$. 
Next, let $\mu^{ \pm}(x, t)$ be eigenvalues of $B_{0}$ satisfying

$$
\mu^{ \pm}(x, t)= \pm \mu(t) \quad \text { at } x=x_{2}(t) .
$$

It follows from (3.9) that $\mu^{ \pm}\left(x_{2}(t), t\right) \rightarrow 0$ as $t \rightarrow t_{0}$, and hence $x_{2}(t)$ is also a turning point of $B_{0}$ at $t=t_{0}$. Then, using the assumption (2.17), we can find a simple turning point $x=x_{1}(t)$ of $B_{0}$ (and hence of $A_{0}$ as well) so that it satisfies

$$
x_{1}(t) \rightarrow x_{2}\left(t_{0}\right) \quad \text { as } t \rightarrow t_{0}
$$

and

$$
\mu^{+}(x, t)=\mu^{-}(x, t) \quad \text { at } x=x_{1}(t) .
$$

Thus the two turning points $x_{1}(t)$ and $x_{2}(t)$ of $A_{0}$ merge at $t=t_{0}$ and, if we let $\lambda^{ \pm}(x, t)$ denote the eigenvalues of $A_{0}$ corresponding to $\mu^{ \pm}(x, t)$ through the relation (3.4), the eigenvalues $\lambda^{ \pm}(x, t)$ of $A_{0}$ merge both at $x_{1}(t)$ and at $x_{2}(t)$. This proves the statement (i) of Theorem 2.1.

We now prove Theorem 2.1, (ii). Proposition 2.3 implies that the two eigenvalues $\nu^{ \pm}(t)$ of $C_{0}$ which tend to 0 as $t \rightarrow t_{0}$ are given by $\pm 2 \mu(t)$. This and (3.11) entail that

$$
\nu^{+}(t)-\nu^{-}(t)=\left.2\left(\mu^{+}(x, t)-\mu^{-}(x, t)\right)\right|_{x=x_{2}(t)} .
$$

Hence, noting that $\lambda^{ \pm}(x, t)$ merge at $x_{j}(t)(j=1,2)$ and that $\mu^{ \pm}(x, t)$ merge at $x_{1}(t)$, we have

$$
\begin{aligned}
\frac{d}{d t} \int_{x_{1}(t)}^{x_{2}(t)}\left(\lambda^{+}-\lambda^{-}\right) d x & =\int_{x_{1}(t)}^{x_{2}(t)} \frac{\partial}{\partial t}\left(\lambda^{+}-\lambda^{-}\right) d x \\
& =\int_{x_{1}(t)}^{x_{2}(t)} \frac{\partial}{\partial x}\left(\mu^{+}-\mu^{-}\right) d x \\
& =\left.\left(\mu^{+}-\mu^{-}\right)\right|_{x=x_{2}(t)} \\
& =\frac{1}{2}\left(\nu^{+}-\nu^{-}\right) .
\end{aligned}
$$

Here we used Proposition 2.2 to derive the second equality. Integrating (3.15) from $t_{0}$ to $t$, we then obtain (2.18). This completes the proof of Theorem 2.1.

Theorem 2.2 is proved in a way similar to that for Theorem 2.1. 
Let $t=t_{0}$ be a turning point of the second kind. Then there exist $\rho_{j}(t)$ $(j=1,2)$ satisfying

$$
h\left(\rho_{j}(t), t\right)=0 \quad \text { and } \quad \rho_{1}(t)-\rho_{2}(t) \rightarrow 0\left(\text { as } t \rightarrow t_{0}\right) .
$$

Let $\mu_{j}(t)$ denote $\sqrt{\rho_{j}(t)}$ and define $x_{j}(t)$ by

$$
g_{\text {even }}\left(\mu_{j}(t), x_{j}(t), t\right)=0 .
$$

Then by the same argument as above we find that $\pm \mu_{j}(t)$ are eigenvalues of $B_{0}$ at $x=x_{j}(t)$ and that $x_{j}(t)$ is a double turning point of $A_{0}$. Furthermore it follows from (3.16) that $x_{1}(t)$ and $x_{2}(t)$ merge at $t=t_{0}$.

If we denote $\mu_{1}\left(t_{0}\right)\left(=\mu_{2}\left(t_{0}\right)\right)$ by $\widehat{\mu}$ and $x_{1}\left(t_{0}\right)\left(=x_{2}\left(t_{0}\right)\right)$ by $\widehat{x}$, we know that $g_{\text {odd }}\left(\mu, t_{0}\right)$ and $g_{\text {even }}\left(\mu, \widehat{x}, t_{0}\right)$ can be divided by $\left(\mu^{2}-\widehat{\mu}^{2}\right)^{2}$ and $\left(\mu^{2}-\widehat{\mu}^{2}\right)$ respectively. Then at $(x, t)=\left(\widehat{x}, t_{0}\right) \pm \widehat{\mu}$ become eigenvalues of $B_{0}$ and they are simple roots of $\operatorname{det}\left(\mu-B_{0}\right)=0$ due to the assumption (2.17). Hence there exist eigenvalues $\mu^{ \pm}(x, t)$ of $B_{0}$ which do not depend on $j$ and satisfy

$$
\mu^{ \pm}(x, t)= \pm \mu_{j}(t) \quad \text { at } x=x_{j}(t) .
$$

Letting $\lambda^{ \pm}(x, t)$ denote the eigenvalues of $A_{0}$ corresponding to $\mu^{ \pm}(x, t)$ through the relation (3.4), we thus find that $\lambda^{ \pm}(x, t)$ merge both at $x_{1}(t)$ and at $x_{2}(t)$.

Finally, Proposition 2.3 again implies that $\pm 2 \mu_{j}(t)(j=1,2)$, denoted by $\nu_{j}^{ \pm}(t)$ in what follows, give the eigenvalues $\nu_{k}^{ \pm}$and $\nu_{k^{\prime}}^{ \pm}$of $C_{0}(t)$ in Theorem 2.2, (ii) and they satisfy the following relations:

$$
\begin{aligned}
\nu_{2}^{+}(t)-\nu_{1}^{+}(t) & =2\left(\mu^{+}\left(x_{2}(t), t\right)-\mu^{+}\left(x_{1}(t), t\right)\right) \\
& =-2\left(\mu^{-}\left(x_{2}(t), t\right)-\mu^{-}\left(x_{1}(t), t\right)\right) .
\end{aligned}
$$

By an argument similar to that employed in deriving (3.15) we then obtain

$$
\begin{aligned}
& \frac{d}{d t} \int_{x_{1}(t)}^{x_{2}(t)}\left(\lambda^{+}-\lambda^{-}\right) d x \\
& =\int_{x_{1}(t)}^{x_{2}(t)} \frac{\partial}{\partial t}\left(\lambda^{+}-\lambda^{-}\right) d x \\
& =\int_{x_{1}(t)}^{x_{2}(t)} \frac{\partial}{\partial x}\left(\mu^{+}-\mu^{-}\right) d x \\
& =\left(\mu^{+}\left(x_{2}(t), t\right)-\mu^{-}\left(x_{2}(t), t\right)\right)-\left(\mu^{+}\left(x_{1}(t), t\right)-\mu^{-}\left(x_{1}(t), t\right)\right) \\
& =\left(\mu^{+}\left(x_{2}(t), t\right)-\mu^{+}\left(x_{1}(t), t\right)\right)-\left(\mu^{-}\left(x_{2}(t), t\right)-\mu^{-}\left(x_{1}(t), t\right)\right) \\
& =\nu_{2}^{+}(t)-\nu_{1}^{+}(t) .
\end{aligned}
$$


This completes the proof of Theorem 2.2.

\section{§4. Proof of Proposition 2.3}

\section{§4.1. In the case of $l=2 m$}

A straightforward computation shows that the top order part $C_{0}$ of the linearized equation of $(N Y)_{2 m}$ at $\widehat{u}$ is of the form

$$
C_{0}=\left(\begin{array}{ccccc}
u_{0}+2 q_{0} & u_{0} & -u_{0} & \cdots & -u_{0} \\
-u_{1} & u_{1}+2 q_{1} & u_{1} & \cdots & u_{1} \\
u_{2} & -u_{2} & u_{2}+2 q_{2} & \cdots & -u_{2} \\
\vdots & \vdots & \vdots & & \vdots \\
u_{2 m} & -u_{2 m} & u_{2 m} & \cdots & u_{2 m}+2 q_{2 m}
\end{array}\right) .
$$

(Here and in what follows every quantity (such as $q_{j}$ ) with the index $j$ running over $1 \leq j \leq N$ is supposed to be cyclic with respect to $j$.) Taking the relations $u_{j}+q_{j}+q_{j+1}=0$ (cf. Lemma 3.1) into account, we then find that

$$
\left(\nu-C_{0}\right)\left(\begin{array}{cccc}
1 & 1 & & \\
& 1 & \ddots & \\
& & \ddots & 1 \\
1 & & & 1
\end{array}\right)=\left(\begin{array}{llll}
\nu-2 q_{0} & \nu+2 q_{1} & & \\
& \nu-2 q_{1} & \ddots & \\
& & \ddots & \nu+2 q_{2 m} \\
\nu+2 q_{0} & & & \nu-2 q_{2 m}
\end{array}\right) .
$$

Hence we obtain

$$
\begin{aligned}
2 \operatorname{det}\left(\nu-C_{0}\right) & =\prod_{j}\left(\nu-2 q_{j}\right)+\prod_{j}\left(\nu+2 q_{j}\right) \\
& =2^{2 m+1}\left[\prod_{j}\left(\frac{\nu}{2}-q_{j}\right)-\prod_{j}\left(-\frac{\nu}{2}-q_{j}\right)\right] \\
& =\left.2^{2 m+2} g_{\text {odd }}(\mu, t)\right|_{\mu=\nu / 2} .
\end{aligned}
$$

This completes the proof of (2.16) in the case of $l=2 m$.

\section{$\S 4.2$. In the case of $l=2 m+1$}

Let us define $\tilde{C}_{0}$ and $\theta$ respectively by $\tilde{C}_{0}=t C_{0} / 2$ and $\theta=t \nu / 2$ so that the following holds:

$$
\operatorname{det}\left(\nu-C_{0}\right)=\operatorname{det}\left(C_{0}-\nu\right)=\left(\frac{2}{t}\right)^{N} \operatorname{det}\left(\tilde{C}_{0}-\theta\right) .
$$


We also introduce new symbols $p_{j}$ and $r_{j}$ defined by

$$
\begin{aligned}
\text { (4.5) } p_{j} & =-t q_{j}=\left[\sum_{r=0 \text { or } 0 \leq s<r \leq m} u_{j-1+2 r} u_{j+2 s}-\sum_{1 \leq r \leq s \leq m} u_{j-1+2 r} u_{j+2 s}\right], \\
r_{j} & =\sum_{1 \leq r \leq s \leq m} u_{j-1+2 r} u_{j+2 s} .
\end{aligned}
$$

Note that the relation

$$
p_{j}+p_{j+1}=t u_{j}
$$

immediately follows from Lemma 3.1. In the case of $l=2 m+1$ (i.e., $N=$ $l+1=2 m+2$ ) each entry of the matrix $\tilde{C}_{0}=\left(M_{i, k}\right)_{0 \leq i, k \leq 2 m+1}$ is then given in terms of these symbols as follows:

$$
\begin{aligned}
M_{i, i} & =r_{i}-r_{i+1}, \\
M_{2 j, 2 l} & =u_{2 j}\left(u_{2 j+1}+u_{2 j+3}+\cdots+u_{2 l-1}-u_{2 l+1}-\cdots-u_{2 j-1}\right)
\end{aligned}
$$

$$
\begin{aligned}
& M_{2 j, 2 l+1}=\left(1-\delta_{l+1, j}\right) u_{2 j}\left(u_{2 l+2}+\cdots+u_{2 j-2}\right) \\
& -\left(1-\delta_{l, j}\right) u_{2 j}\left(u_{2 j+2}+\cdots+u_{2 l}\right) \text {, } \\
& M_{2 j+1,2 l}=\left(1-\delta_{l, j}\right) u_{2 j+1}\left(u_{2 l+1}+\cdots+u_{2 j-1}\right) \\
& -\left(1-\delta_{l, j+1}\right) u_{2 j+1}\left(u_{2 j+3}+\cdots+u_{2 l-1}\right), \\
& M_{2 j+1,2 l+1}=u_{2 j+1}\left(u_{2 j+2}+\cdots+u_{2 l}-u_{2 l+2}-\cdots-u_{2 j}\right) \quad(\text { for } j \neq l) \text {, } \\
& (0 \leq i \leq 2 m+1,0 \leq j \leq m, 0 \leq l \leq m) .
\end{aligned}
$$

Now we first transform the matrix $\tilde{C}_{0}-\theta$ in the following way:

$$
T\left(\tilde{C}_{0}-\theta\right) T^{-1},
$$

where

$$
T=\left(\begin{array}{ccccc|ccc}
1 & & & & & & \\
& & & & & & & \\
& & \ddots & & & & \\
& & & & & & \\
& & & & 1 & & \\
\hline 1 & 0 & 1 & \cdots & 0 & 1 & \\
0 & 1 & 0 & \cdots & 1 & & 1
\end{array}\right)
$$


The matrix (4.8) obtained by this transformation becomes of the form

$$
\left(\begin{array}{ccc|cc} 
& & & * & * \\
& M^{(1)} & & \vdots & \vdots \\
& & & * & * \\
\hline 0 & \cdots & 0 & -\theta & 0 \\
0 & \cdots & 0 & 0 & -\theta
\end{array}\right),
$$

where each entry of the $(2 m) \times(2 m)$ matrix $M^{(1)}$ is given as follows:

$$
\begin{aligned}
M_{j, 2 l}^{(1)} & =2(-1)^{j-1} u_{j}\left(u_{2 l+1}+\cdots+u_{2 m-1}\right) \quad(\text { for } 0 \leq j \leq 2 l-1), \\
M_{2 l, 2 l}^{(1)} & =r_{2 l}-r_{2 l+1}-u_{2 l}\left(u_{2 l+1}+\cdots+u_{2 m-1}-u_{2 m+1}-\cdots-u_{2 l-1}\right)-\theta \\
& =-p_{2 l}+2 u_{2 l}\left(u_{2 m+1}+\cdots+u_{2 l-1}\right)-\theta, \\
M_{j, 2 l}^{(1)} & =2(-1)^{j} u_{j}\left(u_{2 m+1}+\cdots+u_{2 l-1}\right) \quad(\text { for } 2 l+1 \leq j \leq 2 m-1), \\
M_{j, 2 l+1}^{(1)} & =2(-1)^{j} u_{j}\left(u_{2 l+2}+\cdots+u_{2 m}\right) \quad(\text { for } 0 \leq j \leq 2 l), \\
M_{2 l+1,2 l+1}^{(1)} & =r_{2 l+1}-r_{2 l+2}-u_{2 l+1}\left(u_{2 l+2}+\cdots+u_{2 m}-u_{0}-\cdots-u_{2 l}\right)-\theta \\
& =-p_{2 l+1}+2 u_{2 l+1}\left(u_{0}+\cdots+u_{2 l}\right)-\theta, \\
M_{j, 2 l+1}^{(1)} & =2(-1)^{j-1} u_{j}\left(u_{0}+\cdots+u_{2 l}\right) \quad(\text { for } 2 l+2 \leq j \leq 2 m-1)
\end{aligned}
$$

$(0 \leq l \leq m-1)$. Here $\operatorname{det}\left(\tilde{C}_{0}-\theta\right)$ and $\operatorname{det} M^{(1)}$ satisfy the following relation:

$$
\operatorname{det}\left(\tilde{C}_{0}-\theta\right)=\theta^{2} \operatorname{det} M^{(1)} .
$$

Next we transform $M^{(1)}$ by using the following matrices:

$$
\begin{aligned}
& M^{(2)} \stackrel{\text { def }}{=}\left(\begin{array}{c|cc|ccc}
t & & 0 & \\
\hline & 1 & & \\
0 & & \ddots & \\
& & & 1
\end{array}\right)\left(\begin{array}{cccc}
u_{2 m+1} & & 0 & \\
\hline & 1 & & \\
0 & & \ddots & \\
& & & 1
\end{array}\right) \times \\
& \times M^{(1)}\left(\begin{array}{c|cc|cc}
u_{2 m+1}^{-1} & \cdots & \xi_{0, k} & \cdots \\
\hline & 1 & & \\
0 & & \ddots & \\
& & & 1
\end{array}\right)\left(\begin{array}{cc|cc}
t / 2 & & 0 & \\
\hline & & 1 & \\
0 & & \ddots & \\
& & & 1
\end{array}\right)
\end{aligned}
$$


with $\xi_{0, k}(1 \leq k \leq 2 m-1)$ being given by

$$
\begin{aligned}
\xi_{0,2 l} & =-u_{2 m+1}^{-1}\left(u_{2 m+1}+\cdots+u_{2 l-1}\right), \\
\xi_{0,2 l+1} & =u_{2 m+1}^{-1}\left(u_{0}+\cdots+u_{2 l}\right),
\end{aligned}
$$

$(0 \leq l \leq m-1)$. The equality (4.13) entails

$$
\operatorname{det} M^{(2)}=\frac{t^{2}}{2} \operatorname{det} M^{(1)},
$$

and a repeated use of the relation (4.6) verifies the following:

$$
M^{(2)}=\left(\begin{array}{c|ccccc}
M_{0,0}^{(2)} & M_{0,1}^{(2)} & M_{0,2}^{(2)} & M_{0,3}^{(2)} & \ldots & \\
\hline-\left(p_{1}+p_{2}\right) & -\left(\theta+p_{1}\right) & p_{1}+p_{2} & -\left(p_{1}+p_{2}\right) & p_{1}+p_{2} & \ldots \\
p_{2}+p_{3} & & -\left(\theta+p_{2}\right) & p_{2}+p_{3} & -\left(p_{2}+p_{3}\right) & \ldots \\
-\left(p_{3}+p_{4}\right) & & & -\left(\theta+p_{3}\right) & p_{3}+p_{4} & \ldots \\
\vdots & & & & -\left(\theta+p_{4}\right) & \ldots \\
& & & & & \ddots
\end{array}\right),
$$

where

$$
\begin{array}{r}
M_{0,0}^{(2)}=-\left(\theta+p_{0}\right)\left(p_{0}+\cdots+p_{2 m+1}\right)+\left(p_{0}+p_{1}\right)\left(p_{0}+p_{2 m+1}\right), \\
M_{0,2 l}^{(2)}=\left(\theta+p_{0}\right)\left(p_{2 m+1}+p_{0}+\cdots+p_{2 l}\right)-\left(p_{0}+p_{1}\right)\left(p_{0}+p_{2 m+1}\right) \\
(\text { for } 1 \leq l \leq m-1), \\
M_{0,2 l+1}^{(2)}=-\left(\theta+p_{0}\right)\left(p_{0}+p_{1}+\cdots+p_{2 l+1}\right)+\left(p_{0}+p_{1}\right)\left(p_{0}+p_{2 m+1}\right) \\
(\text { for } 0 \leq l \leq m-1) .
\end{array}
$$

We further transform $M^{(2)}$ into

(4.18) $M^{(3)}=M^{(2)}\left(\begin{array}{ccccc}1 & 0 & & & \\ & 1 & 1 & & \\ & & 1 & \ddots & \\ & & & \ddots & 1 \\ & & & & 1\end{array}\right)\left(\begin{array}{cccccc}1 & & & & \\ -1 & 1 & & & \\ 1 & & 1 & & \\ \vdots & & & \ddots & \\ -1 & & & & 1\end{array}\right)\left(\begin{array}{ccccc}0 & & & & -1 \\ 1 & 0 & & & \\ & 1 & 0 & & \\ & & \ddots & \ddots & \\ & & & 1 & 0\end{array}\right)$.

At this step we have

$$
\operatorname{det} M^{(3)}=\operatorname{det} M^{(2)}
$$


and the matrix $M^{(3)}$ thus obtained becomes

$$
M^{(3)}=\left(\begin{array}{cccccc}
M_{0,0}^{(3)} & M_{0,1}^{(3)} & M_{0,2}^{(3)} & \cdots & \ldots & M_{0,2 m-1}^{(3)} \\
-\left(\theta+p_{1}\right) & -\theta+p_{2} & & & & \\
& -\left(\theta+p_{2}\right) & -\theta+p_{3} & & & \\
& & -\left(\theta+p_{3}\right) & -\theta+p_{4} & & \\
& & \ddots & \ddots & \\
& & & -\left(\theta+p_{2 m-1}\right) & -\theta+p_{2 m}
\end{array}\right)
$$

with

$$
\begin{aligned}
& M_{0,0}^{(3)}=-\left(p_{0}+p_{1}\right)\left(\theta-p_{2 m+1}\right), \\
& M_{0, k}^{(3)}=\left(p_{2 m+1}+(-1)^{k+1} p_{k+1}\right)\left(\theta+p_{0}\right) \quad(1 \leq k \leq 2 m-1) .
\end{aligned}
$$

Now let $M^{(4)}$ be a $(2 m+2) \times(2 m+2)$ matrix defined by

$$
\left(\begin{array}{ccccc}
1 & & & & 1 \\
-\left(\theta+p_{0}\right) & p_{0}+p_{1} & & & \\
& -\left(\theta+p_{1}\right) & -\left(\theta-p_{2}\right) & & \\
& & -\left(\theta+p_{2}\right) & -\left(\theta-p_{3}\right) & \\
& & \ddots & \ddots & \\
0 & 0 & -\left(p_{2 m+1}+p_{2}\right)-\left(p_{2 m+1}-p_{3}\right) & \cdots & -\left(\theta-p_{2 m+1}\right)
\end{array}\right) .
$$

Then by a straightforward computation we can readily confirm

$$
\operatorname{det} M^{(4)}=\operatorname{det} M^{(3)} .
$$

Finally, we transform $M^{(4)}$ as follows:

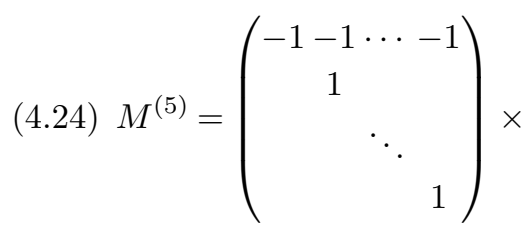

$$
\times\left(\begin{array}{cc|ccc|ccc}
2 \theta & & & & & \\
& 1 & & & & \\
\hline & 1 & & & \\
& & & & \ddots & \\
& & & & & & & \\
& & & -1 & 0 & \cdots &
\end{array}\right) M^{(4)}\left(\begin{array}{cc|cccc}
1 & 1 & & & & \\
& 1 & & & & \\
\hline & & 1 & & & \\
& & & \ddots & & \\
& & & & 1 & \\
& & 1 & \cdots & 1 & 1
\end{array}\right) .
$$


Then

$$
\operatorname{det} M^{(5)}=-2 \theta \operatorname{det} M^{(4)}
$$

holds and the matrix $M^{(5)}$ thus defined becomes

$$
M^{(5)}=\left(\begin{array}{ccccc}
-\left(\theta-p_{0}\right) & & & -\left(\theta+p_{2 m+1}\right) \\
-\left(\theta+p_{0}\right) & -\left(\theta-p_{1}\right) & & & \\
& -\left(\theta+p_{1}\right) & -\left(\theta-p_{2}\right) & & \\
& \ddots & \ddots & \\
& & -\left(\theta+p_{2 m}\right) & -\left(\theta-p_{2 m+1}\right)
\end{array}\right)
$$

The determinant of this matrix is readily computed and we obtain

$$
\operatorname{det} M^{(5)}=\left(\theta-p_{0}\right) \cdots\left(\theta-p_{2 m+1}\right)-\left(\theta+p_{0}\right) \cdots\left(\theta+p_{2 m+1}\right) .
$$

After these long computations, combining the seven equalities (4.4), (4.12), (4.15), (4.19), (4.23), (4.25) and (4.27), and substituting $p_{j}=-t q_{j}$ and $\theta=$ $t \nu / 2=t \mu$, we conclude

$$
\begin{aligned}
(4.28) & \operatorname{det}\left(\nu-C_{0}\right) \\
= & \left.\frac{2^{N}}{t}\left[\mu\left(\left(\mu-q_{0}\right) \cdots\left(\mu-q_{2 m+1}\right)-\left(\mu+q_{0}\right) \cdots\left(\mu+q_{2 m+1}\right)\right)\right]\right|_{\mu=\nu / 2} \\
= & \left.\frac{2^{N+1}}{t}\left[\mu g_{\text {odd }}(\mu, t)\right]\right|_{\mu=\nu / 2} .
\end{aligned}
$$

Since the coefficient of the top degree part of $g_{\text {odd }}(\mu, t)$ is equal to $-\left(q_{1}+\cdots+\right.$ $\left.q_{N}\right)=t / 2$ in this case, this leads to

$$
\operatorname{det}\left(\nu-C_{0}\right)=\left.2^{N} \mu \tilde{g}_{\text {odd }}(\mu, t)\right|_{\mu=\nu / 2} .
$$

The proof of Proposition 2.3 is now completed at last.

\section{$\S 5 . \quad$ Remarks and Open Problems}

As we have observed so far, the Stokes geometry of the Noumi-Yamada system $(N Y)_{l}$ is closely related to the Stokes geometry of its underlying Lax pair. This relationship between the two Stokes geometries is based on the three fundamental facts, i.e., Propositions 2.1, 2.2 and 2.3. We are now conjecturing 
that these three facts do hold (with due modifications) for a quite general Lax pair (1.1). As a matter of fact, similar fundamental facts are confirmed for the $\left(P_{\mathrm{I}}\right)$ and $\left(P_{\mathrm{II}}\right)$ hierarchies in $[\mathrm{KKNT}]$ (where the global aspect of the Stokes geometry of higher-order Painlevé equations is also discussed) and, as is discussed in [T2], Proposition 2.2 is proved in a general situation. (We can also verify Proposition 2.1 in a sufficiently general situation.) However at the present stage it seems difficult to establish Proposition 2.3, i.e., a "bridge" between Painlevé type equations and their underlying Lax pair, in general. It is one of the relevant problems to prove Proposition 2.3 in a general situation.

For traditional Painlevé equations the relations between the two Stokes geometries play a crucially important role in constructing a local reduction to the first Painlevé equation near a simple turning point. We thus believe that the relations of the Stokes geometries established in this paper strongly support the possibility of the exact WKB analysis for the Noumi-Yamada systems, or more generally for higher-order Painlevé equations. Still, in order to develop the WKB analysis for such nonlinear equations, there are many things to discuss. In ending this article, we list up some open problems related to this subject.

$1^{\circ}$ ) How can one construct the general solutions (of WKB type), i.e., solutions containing sufficiently many free parameters, of higher-order Painlevé equations?

$2^{\circ}$ ) As is proved in this paper, the configuration of the Stokes geometry of the Noumi-Yamada system $(N Y)_{l}$ near a simple turning point of the first kind is the same as that of the first Painlevé equation $\left(P_{\mathrm{I}}\right)$ near its unique turning point $t=0$. Then, is it possible to construct a local reduction of $(N Y)_{l}$ to $\left(P_{\mathrm{I}}\right)$ near a simple turning point of the first kind?

$3^{\circ}$ ) What is the "normal form" for higher-order Painlevé equations near a turning point of the second kind?

\section{References}

[AKT] Aoki, T., Kawai, T. and Takei, Y., WKB analysis of Painlevé transcendents with a large parameter. II, in: M. Morimoto, and T. Kawai eds., Structure of Solutions of Differential Equations, World Scientific, (1996), 1-49.

[JMU] Jimbo, M., Miwa, T. and Ueno, K., Monodromy preserving deformation of linear ordinary differential equations with rational coefficients. I, Physica D, 2 (1981), 306-352.

[KKNT] Kawai, T., Koike, T., Nishikawa, Y. and Takei, Y., On the Stokes geometry of higher order Painlevé equations, RIMS Preprint, No. 1443, (2004). 
[KT1] Kawai, T. and Takei, Y., WKB analysis of Painlevé transcendents with a large parameter. I, Adv. Math., 118 (1996), 1-33.

[KT2] , WKB analysis of Painlevé transcendents with a large parameter. III, Adv. Math., 134 (1998), 178-218.

[NY1] Noumi, M. and Yamada, Y., Higher order Painlevé equations of type $A_{l}^{(1)}$, Funkcial Ekvac., 41 (1998), 483-503.

[NY2] - Symmetry in Painlevé equations, in: C. J. Howls, T. Kawai and Y. Takei eds., Toward the Exact WKB Analysis of Differential Equations, Linear or NonLinear, Kyoto Univ. Press, (2000), 245-260.

[T1] Takei, Y., An explicit description of the connection formula for the first Painlevé equation, in: C. J. Howls, T. Kawai and Y. Takei eds., Toward the Exact WKB Analysis of Differential Equations, Linear or Non-Linear, Kyoto Univ. Press, (2000), 271-296.

[T2] $\longrightarrow$, On a double turning point problem for systems of linear ordinary differential equations, Preprint. 